\title{
3D printing of rock analogues in sand: a tool for design and repeatable testing of geomechanical and transport properties
}

\author{
Kevin Hodder ${ }^{1}$, Sergey Ishutov ${ }^{1}$, Angel Sanchez ${ }^{1}$, Gonzalo Zambrano ${ }^{1, *}$, and Rick Chalaturnyk ${ }^{1}$ \\ ${ }^{1}$ University of Alberta, Civil and Environmental Engineering, Edmonton, Alberta, Canada
}

\begin{abstract}
Natural rocks can be heterogeneous due to complex diagenetic processes that affect mineralogy and pore architecture. Correlation of geomechanical and transport properties of rocks in three dimensions can lead to large variances in data when tested experimentally. 3D-printing of rock analogues in sand is a promising alternative for experimental testing that can be used to calibrate variables during geotechnical testing. While 3D-printed sand is a homogeneous material, the parameters for creating grain packing and pore infill can be tuned to mimic specific geomechanical and transport properties. Initially, 3D-printed specimens have a low density due to a loose distribution of grains. Herein, we present our efforts at increasing the density through incorporating a roller in the printing process to compact individual layers. We also propose introduction of a more heterogeneous sand mixture that encompasses a wide range of grain-size distributions. Lastly, a discussion between binder saturation (that infills the pore space) of 3D-printed specimens and the axial strength, dimensional control, and porosity is described within. 3D printing of rock analogues is critical in pursuing rigorous destructive tests required for geotechnical and geological engineering because it can provide repeatable, controlled data on rock properties.
\end{abstract}

\section{Introduction}

Binder jetting is the only additive manufacturing (AM) method capable of fabricating rock analogues from natural sand. In addition to 3D printing with sand [5], [6], several attempts have been made to fabricate rock analogues using gypsum [1], [2] and polymers [3], [4]. Unfortunately, 3D-printed rocks made from gypsum or polymers exhibit elasticity when loaded in compression, which leads to an incorrect material response. 3Dprinted specimens fabricated from sand are ideal for replicating a natural rock, as the specimens are made of more than $90 \%$ of natural material. The remaining $10 \%$ is a mix of polymer binder that acts as an intergranular cement and air. 3D-printed rocks fabricated from sand have been reported extensively [7]-[10], but these studies relied on simple verification of properties using standard printing parameters. Regardless, the use of sand to create test samples for verification of experimental modelling is an unprecedented achievement that integrates geoscience and engineering. 3D-printed rocks provide stakeholders with a tangible, physical specimen that has repeatable mechanical properties, opening the doors for experimental validation. Additionally, the homogenous pore architecture can be used for the study of hydraulic processes, since the same model or sample can be replicated multiple times.

However, previous studies described 3D printing of specimens using factory or default settings, where any deviation of printing parameters was not well understood. An additional drawback of 3D printing with sand is that the inherent porosity is not back filled from millennia of sedimentation, since the specimens can be produced in a manner of hours. Thus, 3D-printed rocks fabricated from sand have a porosity of $\sim 45 \%$ [10], which is higher than in a natural sandstone such as Berea (20-30\% [11]). Increased porosity will result in lower compressive strength and varying hydraulic parameters when flow studies are considered. The increased porosity also makes critical reservoir rock formations consisting of dense carbonate rocks difficult to fabricate.

Several attempts have been made to decrease porosity in 3D-printed powders including external devices that apply a compressive [12] or vibrational [13] force to the powder bed. However, there is no single additive manufacturing or 3D-printing machine that combines sand printing and a densification process. Only two manufacturers of sand 3D printers exist, namely ExOne (USA) and Voxeljet (EU). Unfortunately, both manufacturers utilize a "recoater" that creates individual layers through a hopper, vibration, and gravity. Therefore, a critical material parameter for the sand is flowability, which is a function of particle morphology and size. As the morphology moves to perfect sphericity, the flowability becomes too great and the powder cannot be contained within the recoater. On the opposite extreme, if the particles are too angular, the flowability will be too low resulting in little to no deposition. The same issues occur with particle size, where a particle size below 20 microns results in strong interparticle forces, reducing the sand's flowability.

Powder mixtures have been studied in the metal binder jetting industry, where a broader distribution of

\footnotetext{
* Corresponding author: gonzalo@ualberta.ca
} 
particles would obtain the highest theoretical or "green" density before sintering versus a narrow distribution [14], [15]. To avoid segregation in 3D-printed sand specimens, the sand used by default has a monodisperse and narrow size distribution, with a $\mathrm{D}_{50}$ of 175 microns. A narrow size distribution is used due to the vibrational aspect of deposition, reducing unwanted segregation in finished parts. To reduce the porosity of 3D-printed rocks, sand must be placed into the pore space between larger grains, suggesting that a distribution of particles is inevitable. To avoid segregation, the diameter ratio between the largest and smallest particles can be limited, but this is in direct opposition to densification theory for packed powder beds, where a larger diameter ratio is required [12].

As stated, another possibility of densification has been studied using compressive [12] or vibrational [13] force on the powder bed, directly. By providing vibrational energy into the sand bed, the sand grains will settle into an arrangement that has a higher packing ratio. In all cases, these forces must be implemented externally and should not be a part of the 3D-printer hardware. The effect of changing printing parameters during fabrication of 3D-printed rocks is generally unknown at this time. For this reason, we limited our study to possibilities that could be tested with ease, without any complicated changes to existing equipment. We proposed adjusting the diameter of the metal powder roller that contacts the powder bed after each layer, so that it compacts the sand instead of merely smoothing the layer. This study also investigates how the contact area, which is a function of the radius of the roller, affects the density of 3D-printed rocks. In parallel, we explored the effects of introducing different distributions of sand composed of coarse ( $\mathrm{D}_{50}=175$ microns) and fine $\left(D_{50}=105\right.$ microns) grains, with the attempt at reducing intergranular porosity of the coarser grains.

\section{Experimental Procedure}

\subsection{Materials}

The materials required for $3 \mathrm{D}$ printing were purchased from the manufacturer (ExOne, USA) including the coarse silica sand $\left(D_{50}=175\right.$ microns $)$. A fine sand with a $\mathrm{D}_{50}$ of 105 microns was purchased from Badger Cast (USA). Both sand types were composed of $\sim 99 \%$ silica (quartz). Five sand mixtures were used in each set (Table 1) to test repeatability of morphometric features (e.g., volume, diameter, shape), porosity (total and effective), and geomechanical properties (compressive strength). The $\mathrm{D}_{50}$ for the coarse and fine sands were obtained by sieving.
Table 1: The different grades of sand mixtures used to 3D print specimens from sand.

\begin{tabular}{ccc} 
Mixture & $\begin{array}{c}\text { Coarse Sand } \\
\left(\mathrm{D}_{50}=175 \mu \mathrm{m}\right)\end{array}$ & $\begin{array}{c}\text { Fine Sand } \\
\left(\mathrm{D}_{50}=105 \mu \mathrm{m}\right)\end{array}$ \\
\hline $100 \mathrm{C}$ & $100 \%$ & $0 \%$ \\
$75 \mathrm{C} / 25 \mathrm{~F}$ & $75 \%$ & $25 \%$ \\
$50 \mathrm{C} / 50 \mathrm{~F}$ & $50 \%$ & $50 \%$ \\
$25 \mathrm{C} / 75 \mathrm{~F}$ & $25 \%$ & $75 \%$ \\
$100 \mathrm{~F}$ & $0 \%$ & $100 \%$
\end{tabular}

Two rollers were tested on the M-Flex printer for compaction of sand layers after deposition by the hopper (Fig. 1). The first roller was purchased from ExOne as part of the M-Flex 3D printer. The first roller had a diameter of $29 \mathrm{~mm}$ and is henceforth defined as the "small roller". The second roller, manufactured at the Reservoir Geomechanics Research Group (University of Alberta, Edmonton, Canada), was $48 \mathrm{~mm}$ in diameter, henceforth defined as the "large roller". Both rollers were made from aluminum and hard anodized. Binder saturation was fixed at $20 \%$ for all the samples. Binder saturation was one of the most important parameters controlling the mechanical behavior of the rock analogue and has been studied elsewhere [7]-[10], [16].

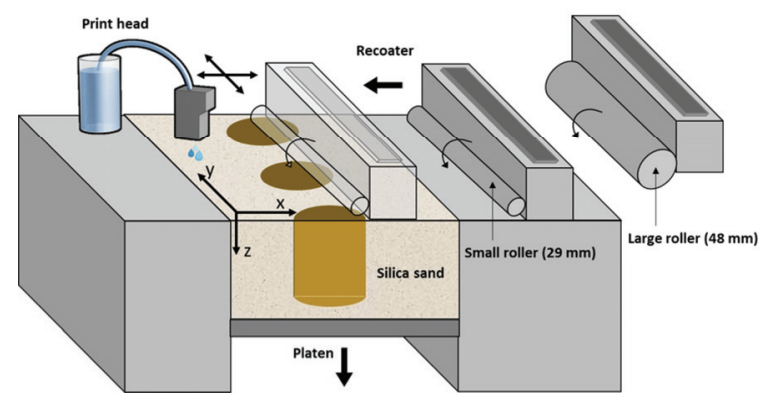

Fig. 1. Illustration of the 3D-printing area indicating the large and small rollers used for fabrication of specimens.

\subsection{D Printing and Post-processing}

An extensive description of the $3 \mathrm{D}$ printing process can be found elsewhere [7], [10]. A summary is provided as follows: three different sizes of cylindrical specimens were printed for each powder mixture to perform experimental analyses: 1) $63.5 \times 127 \mathrm{~mm}$; 2) $38.1 \times 76.2$ $\mathrm{mm}$ and 3) $12.7 \times 25.4 \mathrm{~mm}$. The M-Flex printer (ExOne) was used to fabricate all analogue sandstones. The overall printing process involved three core steps: 1) mixing $3.5 \mathrm{~kg}$ of silica sand with $5 \mathrm{~mL}$ of p-toluene sulphonic acid (activator) for approximately 2 minutes to coat the sand grains. The acid-coated sand was then added to a hopper at the top of the M-Flex printer; 2) the hopper then deposited the sand into a vibrating spreader 
(recoater) that moved along the $x$-axis parallel to the job box (Fig. 1) spreading a $400-\mu \mathrm{m}$ thick layer of silica sand. Following the recoater, the roller was used to even out and compact the sand layer; 3 ) finally, the print head jetted furfuryl alcohol (binder) onto the sand layer while moving along the $x-y$ axis, following the pattern of the digital file pre-loaded onto the M-Flex computer. The post-printing process was necessary for achieving full strength of the specimens and consisted of curing the $3 \mathrm{D}$-printed samples in a large oven at $80^{\circ} \mathrm{C}$ for 24 hours. This step helped reduce the moisture in the samples from the polymerization reaction between the binder and the activator [7], [10]. The process described above was repeated for both rollers. After the curing process, the samples were cleaned to remove the loose sand from the unbounded regions. Finally, the samples were measured with a caliper and weighed to acquire an estimate of their density.

\subsection{CT Scanning}

Smaller samples $(12.7 \times 25.4 \mathrm{~mm})$ were CT (computed tomography) scanned using SkyScan 1172 (Bruker), with $60 \mathrm{keV}$, at a resolution of 17 microns per voxel. An aluminum filter was used to reduce the noise and scanning artifacts. Each sample was reconstructed to a full 3D volume in the CTAn software (Bruker) to calculate porosity. A threshold was applied to the CT images to segment the pore and solid phases. The solid phase comprised the combination of sand grains and binder due to insufficient resolution of the CT images to separate the grayscale intensities for grains and binder. The porosity was calculated on segmented images as the ratio of black voxels (pores) to the total number of voxels. Scanning time for each sample was $\sim 2$ hours.

\subsection{Helium Pycnometry}

CT-scanned samples were used to identify the solid volume and calculate the helium porosity. The machine for helium pycnometry was a Quantachrome Pentapyc 5200e. A cell of $10 \mathrm{~cm}^{3}$ was used for this study. The gas was ultra-high purity helium from Praxair (99.9\%). Because the sample had a known mass, it was placed in one of two chambers of known volume and maintained at a constant temperature, followed by addition of helium. The resulting equilibrium pressures were used in conjunction with the ideal gas law to determine the apparent density of the solid phase (grains and cured binder).

\subsection{Unconfined Compressive Strength (UCS) Testing}

An Instron $400 \mathrm{kN}$ system was used to obtain the UCS value for the different analogue sandstones. The specimen size for all the tests had a height and diameter of $127 \mathrm{~mm}$ and $63.5 \mathrm{~mm}$, respectively, maintaining the ASTM recommended height/diameter ratio of 2:1.
Moreover, all samples were printed with a fixed bedding orientation (all samples were 3D-printed horizontally).

\section{Results and Discussion}

\subsection{Morphometric Features}

3D-printed sandstone analogues allow us to reduce specimen-to-specimen heterogeneity, providing accurate control over the explicit inclusions, such as fracture networks, porosity, grain size distribution, and density distribution. By knowing and controlling inter-sample variability, 3D printing provides a valuable tool to validate numerical geomechanical models. For this work, the volumes of 3D-printed sandstones and its corresponding digital volumes were compared. There were no significant morphological changes, and the standard deviation (SD) between the 3D-printed and the digital volume was $1.33 \%(n=5)$ for the small roller and $4.88 \%(n=5)$ for the large roller. A higher SD for the larger roller suggests that the higher compaction may provide slight variation in density.

CT images allowed us to visualize an internal texture of 3D-printed samples and assess the consistency in geometry of the top and bottom of each sample (Fig. 2). Specimens printed with the small roller showed more deficiencies in the shape of the cylinder, especially in the diameter. The walls of the cylindrical samples were also not perfectly vertical. These inconsistencies might arise from micro-scale shifting of grains within the layer upon movement of the roller and binder jetting. Because the samples were printed horizontally in regard to the cylinder height, the highest accumulation of pores could be observed between the layers, where the pore connectivity was formed. Specimens 3D-printed with the large roller had more accurate walls than diameters, which might be a result of higher compaction on individual layers.

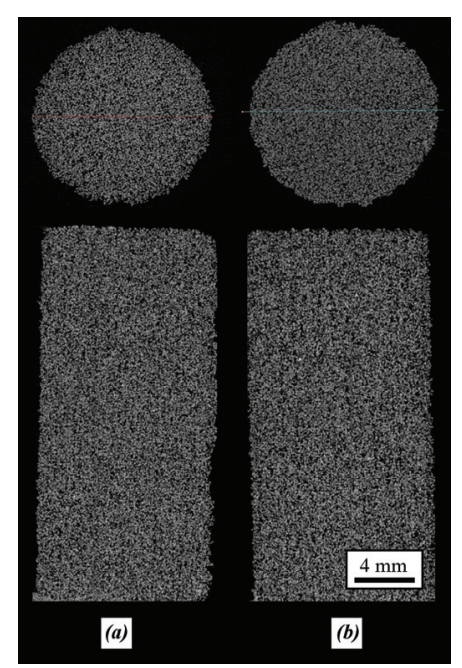

Fig. 2. CT images of specimens 3D-printed in coarse sand. (A) Top view; (B) Profile view. Left image - small roller, right image - large roller. 


\subsection{Porosity}

Porosity is one of the key parameters that is used to evaluate transport properties in porous media. For both natural and 3D-printed sandstones, porosity can indicate the amount of pore space in the bulk volume. CT images form a stack that is used for the total porosity calculation as the ratio of segmented voxels to the total number of voxels in a given volume of a specimen. A more valuable property is effective or connected porosity that is calculated from helium pycnometry tests. For 3Dprinted samples, as well as for natural rocks, CT porosity can be higher than the helium porosity due to closed porosity (where the helium cannot penetrate). An explicit trend of clustered porosity is observed for both sets of 3D-printed specimens (Fig. 3). Samples printed with the small roller show a higher range of variation among CT and helium porosity. Specimens fabricated with a higher percentage of fine sand were observed to have a higher porosity than specimens printed with a majority of coarse sand.

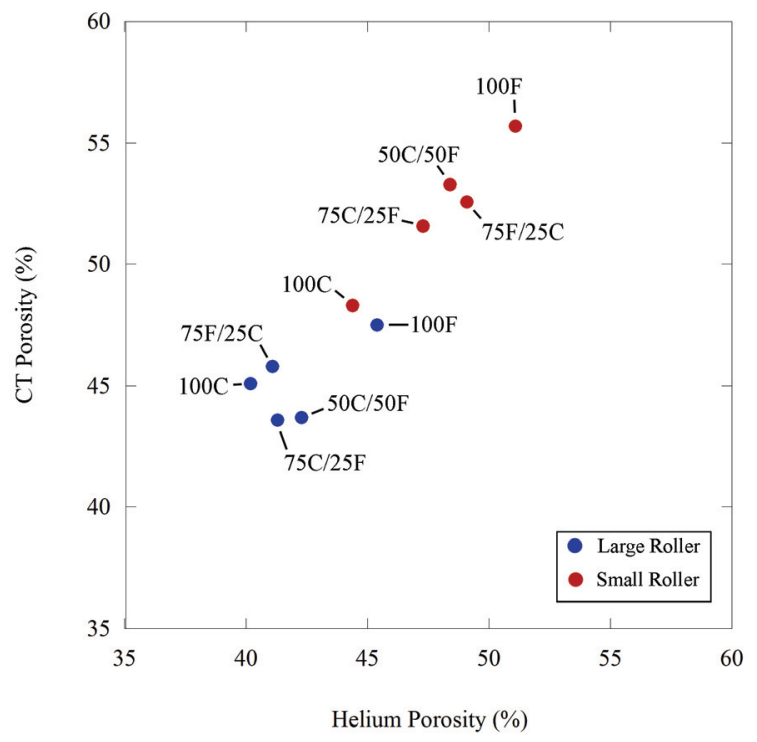

Fig. 3. A graphical representation of $\mathrm{CT}$ and helium pycnometry measurements, where samples printed with the small roller show a greater variation.

Between the two rollers, the large roller is more effective for reducing porosity in sand layers during 3D printing (Fig. 4). In terms of sand mixtures, as fines are introduced, the porosity begins to increase. This is consistent for helium-CT porosity (Fig. 3), which indicates a higher number of connected pores within the total pore volume. It is suggested that the greater compaction of sand by the large roller is due to its larger mass and radius of the curvature that can apply higher pressures during $3 \mathrm{D}$ printing. However, the fine sand has a unimodal particle size distribution, which is not efficient for particle packing. The coarse sand size distribution has a broader distribution, meaning there is a greater number of finer particles ( $<100$ microns) in this sand that contrast the larger particles, providing more efficient packing. This suggestion is in line with random packing, whereas the diameter ratio between the particles increases, the density increases [12]. Therefore, a higher ratio between the smallest and the largest grains in the mixture should result in a more heterogeneous distribution of particles within the pore space. This phenomenon is similar to grain sorting in natural materials, where moderately or poorly sorted rocks contain a higher proportion of fine-to-coarse particles and can result in lower porosity. Combined with the roller surface area, which affects the amount of stress applied to the sand layer, 3D-printed rocks containing a broader distribution of particles are shown to maintain a higher density. The variance in density for all mixtures is noticeable from the porosity reduction, but the bulk density measured by helium pycnometry only decreased by up to $0.07 \mathrm{~g} / \mathrm{cm}^{3}$. The $100 \mathrm{C}$ sample shows the highest densification (Fig. 4), while the $50 \mathrm{~F} / 50 \mathrm{C}$ sample has the least. Interestingly, the $75 \mathrm{C} / 25 \mathrm{~F}$ mixture has the highest increase in density, which aligns with spherical packing theory outlining $\sim 21 \%$ of fines being optimum for densification [12].

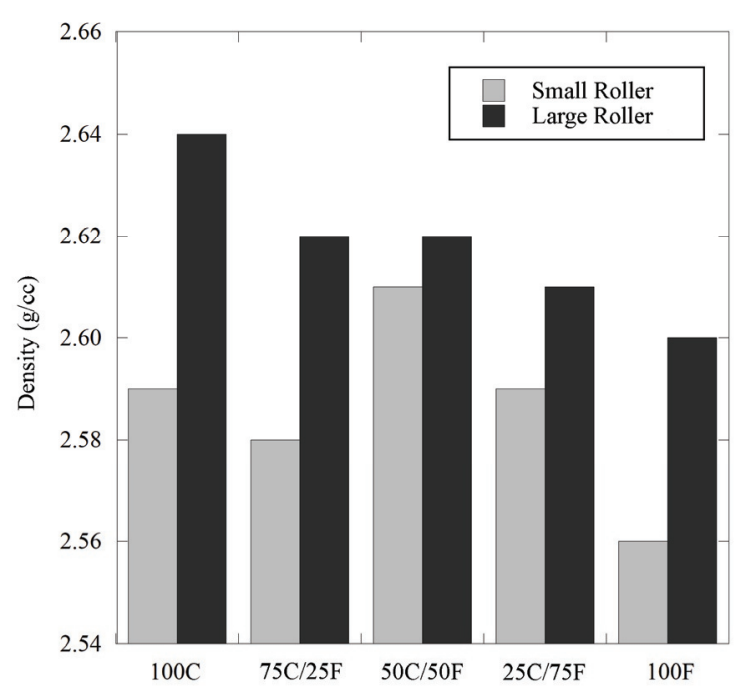

Fig. 4. Comparison of density between samples 3D-printed with the small roller and the large roller. Density was measured using helium pycnometry.

\subsection{Mechanical Strength}

A summary of the UCS test results (Fig. 5) shows the UCS recorded at a range between 15 and $28 \mathrm{MPa}$ for all samples. The comparison of the UCS value for the 3Dprinted analogues with its respective roller indicates that the highest strength difference is observed for the coarse sand (21.25 to $28.05 \mathrm{MPa}, \pm 6.8 \mathrm{MPa})$. Other mixtures have less variability in UCS (14.57 to $19.99 \mathrm{MPa}, \pm 0.7$ to 3.42) $\mathrm{MPa}$, with the larger roller providing an increase in strength. Due to the large difference in strength for the coarse sand, it is possible that an extraneous variable can be causing a decrease in this strength. This suggestion is supported by the higher density achieved when analyzed via helium pycnometry (Fig. 3). 
The failure mode was characterized by a cone-shaped block that formed at one end of the specimen and axial splitting from the apex of the cone (Fig. 6). Figure 7 provides graphs of the compressive stress versus axial strain for all UCS tests. As mentioned above, compressive strengths were recorded at a maximum of $28 \mathrm{MPa}$ for the specimen 3D-printed with the coarse sand and the small roller. The minimum strength was recorded at $15 \mathrm{MPa}$ for the specimen 3D-printed with the fine sand and the small roller. Axial strain at failure was observed at $1.81 \%$ and $1.03 \%$, respectively. All specimens failed in a brittle, catastrophic fashion.

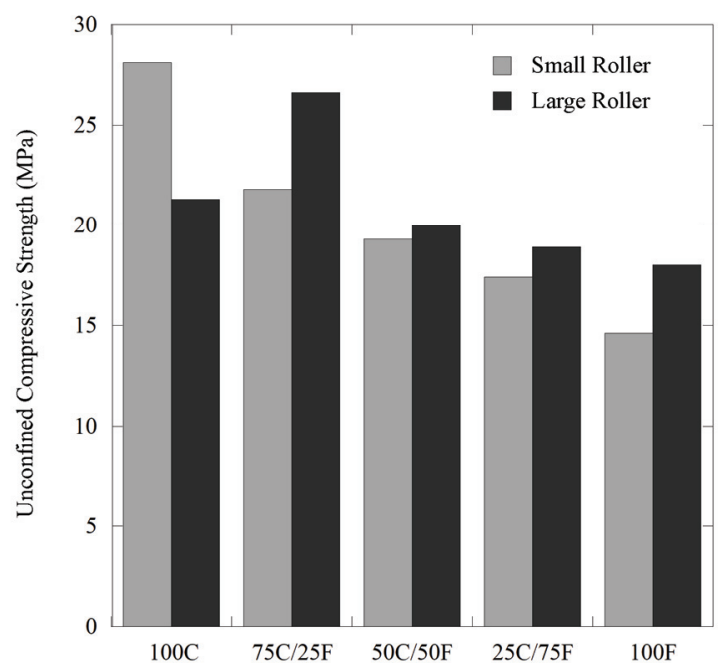

Fig. 5. Comparison of the UCS between the samples printed with the small roller and the large roller, respectively.

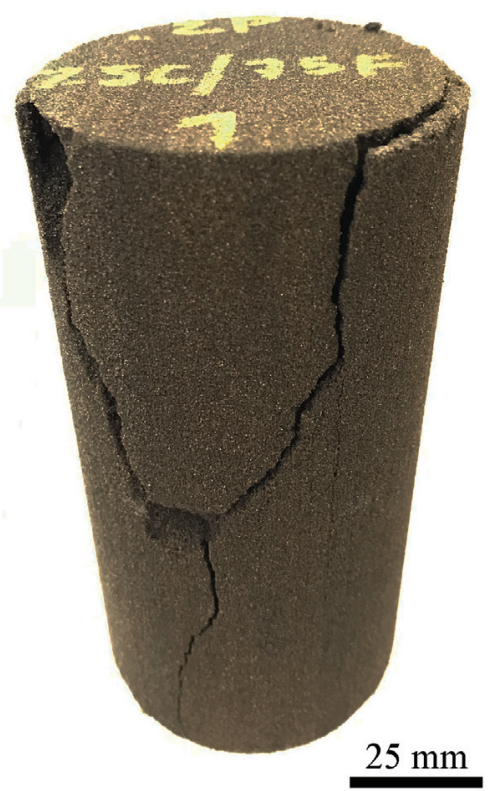

Fig. 6. Characteristic failure mode for all the samples 3Dprinted with both types of rollers.
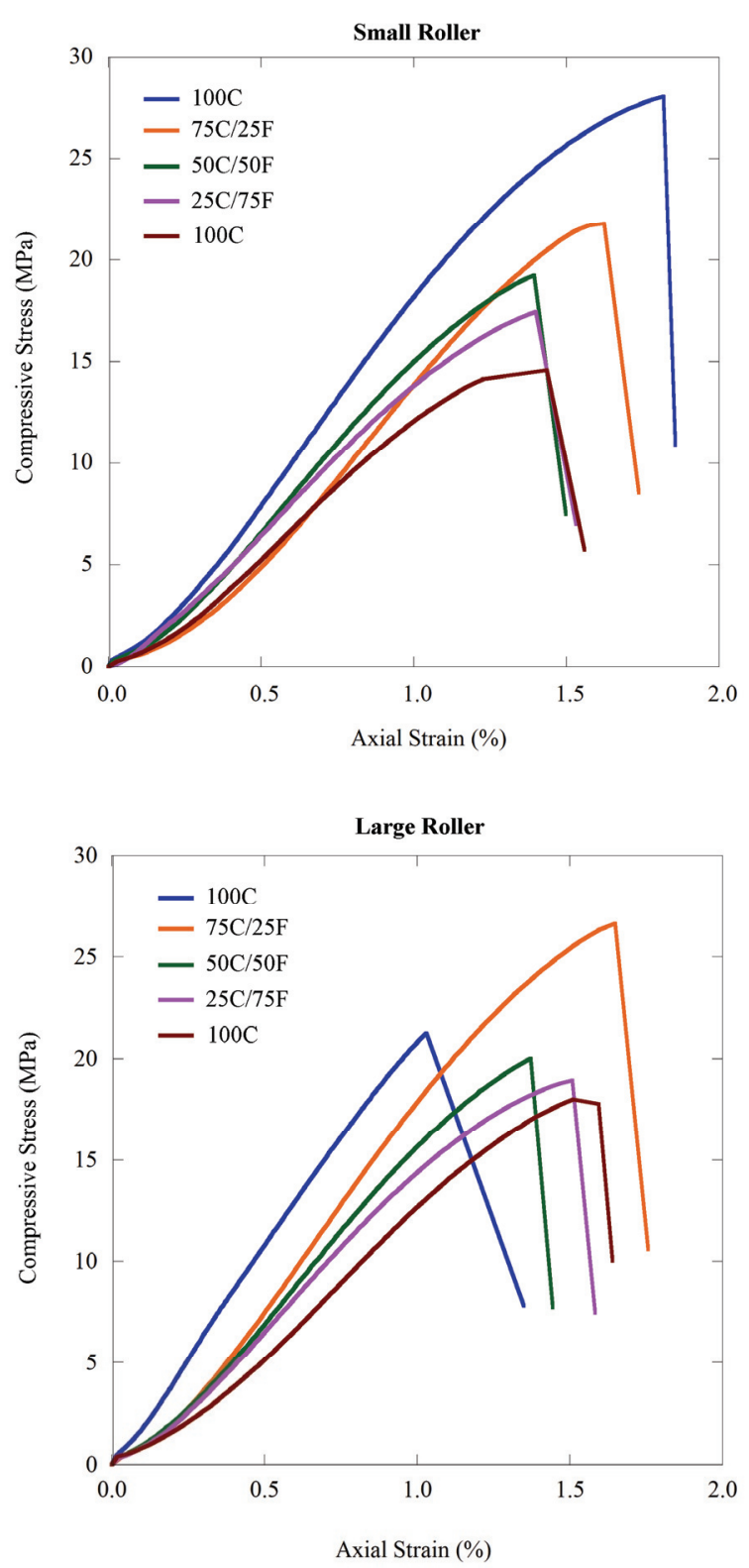

Fig. 7. Comparison of the UCS between the samples printed with the small and large roller, respectively.

\section{Conclusions}

The effects of varying sand grade distributions and roller diameter were observed using UCS testing, helium pycnometry, CT scanning and weight changes. It was found that the large roller achieved a lower porosity overall in the $3 \mathrm{D}$-printed specimens, by up to $6 \%$ across all sand mixtures. Introduction of smaller sand particles resulted in higher porosity and lower strength overall, with the worst case represented by the mixture containing $100 \%$ fine sand. Because the binder saturation was kept constant throughout fabrication, the 
finer sand introduced more surface area, which might have resulted in the binder being spread over a larger area. Due to spreading, the binder necks would be smaller and weaker, resulting in weaker and more porous rock analogues. To combat this issue, a fundamental relationship must be determined between binder saturation and binder equilibrium. Future work includes establishing this relationship, along with introduction of even finer sand to achieve a higher particle diameter ratio. The current study resulted in a particle diameter ratio of 1.5 , which was in disagreement with the ratio of higher than 7 as suggested in the literature. Since this particle diameter ratio would result in a fine particle size of $\sim 21$ microns, a move to coarser grains may be required to avoid interparticle forces and a reduction in flowability. In addition, we will explore the relationship between the mechanical strength and location of samples in the job box, while continuing to work on governing principles surrounding binder jetting additive manufacturing.

\section{References}

[1] L. Kong, M. Ostadhassan, C. Li, N. Tamimi, J., "Pore characterization of 3D-printed gypsum rocks: a comprehensive approach", Mat. Sci., 53, 5063-5078 (2018).

[2] C. Jiang, G.F. Zhao, J.B. Zhu, Y.X. Zhao, L. Shen, "Investigation of dynamic crack coalescence using a gypsum-like 3D printing material", Rock Mech. Rock Eng., 49, 39833998 (2016).

[3] J. Glasschroeder, E. Prager, M.F. Zaeh, "Powder-bed-based 3D-printing of function integrated parts", Rapid Prototyp. J., 21, 207215 (2015).

[4] L. Huang, R.R. Stewart, N. Dyaur, J. BaezFranceschi, "3D-printed rock models: Elastic properties and the effects of penny-shaped inclusions with fluid substitution", Geophys., 81, 669-677 (2016).

[5] S. Fereshtenejad J.J. Song, "Fundamental study on applicability of powder-based 3D printer for physical modeling in rock mechanics", Rock Mech. Rock Eng., 49 (2016).

[6] D. Vogler, S.D.C. Walsh, E. Dombrovski, M.A. Perras, "A comparison of tensile failure in 3Dprinted and natural sandstone", Eng. Geol., 226, 221-235 (2017).

[7] K.J. Hodder, J.A. Nychka, R.J. Chalaturnyk, "Process limitations of 3D printing model rock", Prog. Addit. Manuf., 3, 173-182 (2018).

[8] J.S. Gomez, R.J. Chalaturnyk, G. ZambranoNarvaez, "Experimental Investigation of the Mechanical Behavior and Permeability of 3D Printed Sandstone Analogues Under Triaxial Conditions", Transp. Porous Media, 129, 541557 (2018).

[9] S. Osinga, G. Zambrano-Narvaez, R. Chalaturnyk, "Study of geomechanical properties of 3D printed sandstone analogue", Proceed. Amer. Rock Mech. Assoc., ARMA 15547 (2015).

[10] B. Primkulov, J. Chalaturnyk, R. Chalaturnyk, G.Z. Narvaez, "3D printed sandstone strength: curing of furfuryl alcohol resin-based sandstones", 3D Print. Addit. Manuf., 4, 149155 (2017).

[11] P. Churcher, P. French, J. Shaw, "Rock properties of berea sandstone, baker dolomite, and indiana limestone", Soc. Pet. Eng., 21044: 431-440 (1991).

[12] S. Gregorski, "High green density metal parts by vibrational compaction of dry powder in the three dimensional printing process", $\mathrm{PhD}$ Thesis, MIT, US (1996).

[13] J. Ayer. F. Soppet, "Vibratory compaction: ii, compaction of angular shapes", J. Am. Ceram. Soc., 49, 207-210 (1966).

[14] Y. Bai, G. Wagner, C. Williams, "Effect of particle size distribution on powder packing and sintering in binder jetting additive manufacturing of metals", J. Manuf. Sci. Eng., 139, 081019 (2017).

[15] A. Mostafaei, P. Rodriguez De Vecchis, P. Nettleship, M. Chmielus, "Effect of powder size distribution on densification and microstructural evolution of binder-jet 3d printed alloy 625", Mater. Des. 162, 375-383 (2019).

[16] N. Ardila, G. Zambrano-Narvaez, R.J. Chalaturnyk, "Wettability measurements on $3 \mathrm{~d}$ printed sandstone analogues and its implications for fluid transport phenomena", Transp. Porous Media, 129, 521-539 (2018). 